\title{
La oralidad y la escritura en el proceso de aprendizaje Aplicación del método aprende a escuchar, pensar y escribir
}

\section{Elvira Ivone \\ González Jaimes \\ Universidad Autónoma del Estado del México \\ ivonegj@hotmail.com}

\section{María de Lourdes}

Hernández Prieto

Universidad Autónoma del Estado del México

lulahp@yahoo.com.mx

Juan Márquez Zea

Universidad Autónoma del Estado del México jmarquez03@hotmail.com

\section{Resumen}

En este trabajo presentamos una investigación de estudiantes con rezago o irregularidad estudiantil con cuatro evaluaciones a través del método aprende a escuchar pensar escribir (AEPE). La población estudiada fue de 170 estudiantes, divididos en dos grupos (uno control y otro experimental), de los dos géneros de las licenciaturas de Contaduría y Administración de Empresas. Se emplearon los test de matrices progresivas de Raven, test de estrategias de aprendizaje ACRA y trayectorias académicas. Los resultados muestran que el impacto obtenido por la intervención, AEPE, fue de $23 \%$, con lo que se observa una diferencia significativa en una evaluación de seguimiento de seis y doce meses. En cuanto a la mejora de habilidades de aprendizaje en la medición postest se observó un aumento del $28 \%$ en los promedios generales. Asimismo, hubo una disminución de materias reprobadas en los dos grupos en evolución (doce meses), en el grupo control fue de 3 a 4 y en el grupo experimental fue de 1 a 2 .

Palabras clave: irregularidad estudiantil, oralidad, escritura, comprensión. 


\title{
Orality and writing, students learning processes. Application of the method learn to listen think write
}

\begin{abstract}
Research design is quasi-experimental, field, with four longitudinal assessments for two groups (one experimental and one control) of students with irregularity student. Intervention, learns listen-think-write (lltw) method. population, 488 students. With a sample conditioned (students with irregular registration) of 170 students from two university careers of accounting and administration, both manner, 24.3 years old. Material, Raven's Progressive Matrices test and Learning strategies ACRA test. The impact obtained by the intervention (LLTW) was 23\%, significant difference between follow-up evaluation 6 and 12 months. Regarding the improvement of learning skills in post-test measurement showed a general increase in average by $28 \%$. Also it is necessary to say, the decline was failed subjects in the two groups evolving 12 months in control group was 3 to 4 and the experimental group was 1 to 2 .
\end{abstract}

Keywords: irregular registration, orality, writing, understanding.

\section{Introducción}

Existen problemas de rendimiento académico que afectan la calidad de la educación en las instituciones de educación superior en diversos escenarios universitarios mundiales; éstos se ven reflejados en indicadores como son: el rezago ${ }^{1}$ o irregularidad estudiantil, ${ }^{2}$ lo cual influye directamente tanto en la eficiencia terminal, ${ }^{3}$ como en la obtención del título o grado. Prueba de ello son algunos de los estudios recientemente hechos por Buela-Casal y Castro (2008) en los que analizan los criterios y estándares con los que evalúan los programas educativos en función de la mención de calidad educativa para disminuir los índices de irregularidad estudiantil. Por otro lado, el estudio de Roales (2007) se centra en el análisis de la movilidad de alumnos, mientras que en el artículo de Moyano, Delgado y Buela-Casal (2006) se evalúa la productividad científica en programas de apoyo educativo que favorecen la eficiencia terminal en España a través de las tesis doctorales.

\footnotetext{
${ }^{1}$ Por rezago se entiende el atraso en la inscripción a las asignaturas subsecuentes del plan de estudios al término de un periodo lectivo (Programas Institucionales de Tutoría, 2002).

${ }^{2}$ La irregularidad estudiantil es un concepto similar al rezago utilizado en la Coordinación de Servicios Escolares, Plantel Centro Universitario del Valle de México (2010).

${ }^{3}$ Eficiencia terminal es la relación cuantitativa entre los alumnos que ingresan y los que egresan de una misma cohorte (Programas Institucionales de Tutoría, 2002).
} 
Un estudio realizado por Díaz de Cossio en 1998 para la Asociación Nacional de Universidades e Instituciones de Educación Superior (ANUIES) expone cifras nacionales, en las que se afirma "que de cada 100 estudiantes que inician estudios de licenciatura, entre 50 y 60, concluyen las materias del plan de estudios cinco años después y, de éstos, tan sólo 20 obtienen título. De los que se titulan, solamente un $10 \%$, [sic] es decir 2 egresados, lo hacen a la edad considerada como deseable (24 o 25 años); los demás lo hacen entre los 27 y los 60 años".

Por su parte, la Universidad Veracruzana indica que " 25 de cada 100 estudiantes que ingresan al nivel universitario abandonan sus estudios, la deserción alcanza al 36\% de quienes ingresaron, cifra que se incrementa semestre con semestre, hasta alcanzar el $46 \%$ al término del periodo de formación considerado" (Chain, 1999).

Con base en los anteriores indicadores podemos observar que lamentablemente el problema no ha disminuido en la actualidad. Es por ello, que nuestro trabajo realiza un acercamiento al problema de la irregularidad estudiantil, base de la deserción, así como la baja eficiencia terminal y la escasa obtención de grado. Para la investigación se toma un método de apoyo educativo centrado en la oralidad y en la escritura, como competencias comunicativas claves para disminuir de forma significativa la irregularidad estudiantil.

Cabe aclarar que consideramos las licenciaturas de Contaduría y Administración porque deseamos incrementar la eficiencia terminal, ya que estas licenciaturas han sido avaladas por el Consejo de Acreditación en la Enseñanza de la Contaduría y Administración (CACECA), por lo cual es importante elevar el estándar educativo de las mismas. En este marco se solicitó el apoyo del Programa Institucional de Tutoría Académica (ProInsta), cuyo objetivo es elevar la eficiencia terminal; para lograrlo aplicamos diversas estrategias con el fin de disminuir los índices de irregularidad estudiantil.

De acuerdo con ProInsta (corte junio 2009), las licenciaturas de Contaduría y Administración de Empresas presentan los siguientes datos: alumnos inscritos 488, alumnos irregulares 170 (34.83\%), deserción 36 (7.37\%). Ahora bien, de los es-

${ }^{4}$ Los datos se obtuvieron del Departamento de Control Escolar, Coordinación de Servicios Escolares del plantel Centro Universitario del Valle de México (2010). 
tudiantes irregulares, el $18.03 \%$ tienen un promedio semestral que fluctúa entre el 5.0 y 6.9 (la calificación aprobatoria por materia es de seis) con aproximadamente tres o más materias reprobadas, ${ }^{5}$ quienes se encuentran en inminente riesgo de una posible deserción escolar o baja reglamentaria; por un lado, orillados por las normas disciplinarias de la escuela; y, por otro, por la baja motivación que tienen los estudiantes para ver su bajo aprovechamiento escolar.

En este trabajo se aplican las técnicas específicas de aprendizaje del método aprende a escuchar pensar escribir (AEPE) porque integra los elementos cognitivos requeridos por el perfil de egreso de los estudiantes de las carreras de Contaduría y Administración para elevar el rendimiento académico. El método se encuentra basado en la oralidad y la escritura, elementos básicos para elevar el conocimiento y la cultura (Allgood, Risko, Álvarez y Fairbanks, 2000; y Pintrich, 2004).

Carlos Paul y Ángel Vargas en su artículo "México inmerso en el analfabetismo funcional, una catástrofe silenciosa" (La Jornada, 2001, pp. 1-2) nos mencionan que en un estudio realizado por la UNESCO, México ocupa el penúltimo lugar mundial de 108 países evaluados con un promedio de 2\% de la población que cuenta con hábitos permanentes de lectura (Gutiérrez y Montes de Oca, 2004), fenómeno que no ha disminuido en la última década. Por su parte, la encuesta nacional de hábitos, prácticas y culturas de consumo cultural 2010, presentada por Conaculta, arroja resultados preocupantes en el terreno de la lectura: en México sólo 27\% leyó un libro en los últimos 12 meses, más grave aún es el hecho de que los mexicanos que dijeron haber leído en ese periodo sólo $13 \%$ terminó la lectura; otro dato preocupante es que en un país de 120 millones de habitantes, donde más de $90 \%$ de la población ha recibido formación escolar, $43 \%$ no conoce una biblioteca. Aunado a ello se encuentra el hecho de que la mayoría de los escritos académicos que entregan los alumnos o las tesis de grado no se diferencian unas de otras en cuanto a la expresión escrita, sin importar la disciplina y el tema que traten. Los profesores en el aula invitan a la lectura, pero se niegan a incentivar la reflexión sobre lo leído y se conforman con solicitar resúmenes o cuestionarios que no hacen más que repetir el contenido de la lectura, actividades que adormecen el cerebro y le niegan la posibilidad de pensar y, por tanto, niegan la oportunidad de escribir a sus estudiantes (Hauy, 2008).

${ }^{5}$ Idem. 
La mayor parte de los académicos de todos los niveles piden a sus alumnos que elaboren textos escritos; sin embargo, no hay un proceso para reflexionar, criticar, ponderar y analizar las lecturas que realizan los estudiantes, con lo que les niegan la posibilidad de incentivar otras competencias de escritura. La escuela ha pretendido introducir la lectura como elemento obligatorio (castigo) y no como una actividad placentera (Argüelles, 2007). El alumno es evaluado a partir de que contesta preguntas de forma mecánica sobre el texto y no hace del texto un pretexto para el uso del ocio como goce, ni hace del texto un pretexto para la escritura o la oralidad.

Lo anterior, quizá, se deba a que el docente no conceptualiza los actos del habla (leer, escribir, hablar y escuchar) como una destreza esencial de toda actividad humana. ${ }^{6}$ De estas habilidades comunicativas a la que menos importancia le da la escuela es la oralidad (hablar y escuchar, algo "natural" al ser humano), tarea que desarrollamos durante toda la vida. Sin embargo, no todas las manifestaciones comunicativas orales son "naturales", pues algunas requieren ser aprendidas con el mismo grado de seriedad, calidad y complejidad que exige, por ejemplo, la escritura o la lectura; en algunas de esas manifestaciones se encuentran la conferencia, la exposición en clase, la charla, la entrevista, el debate y el diálogo académico (Donolo Chiecher y Rinaudo, 2004).

En la oralidad - igual que en las otras competencias comunicativas - se perfilan perfectamente dos procesos: la emisión (hablar) y la recepción (escuchar). La oralidad es la primera herramienta del pensamiento que contribuye a designar el mundo real y el mundo ficticio, lo que se ve y lo que no ve, lo específico y lo simbólico. Por tanto, con la oralidad es posible crear y re-crear seres, formas y significados. Así, la oralidad interna (cuando uno se escucha a sí mismo) y la oralidad externa (cuando construye ideas o pensamientos para que el otro lo escuche) son concebidas en su función fundadora que da vida a la especie; esto es, como creadora de la humanidad (Reyzábal, 2006).

\footnotetext{
${ }^{6}$ Sin embargo, los planes y programas de estudio de educación media superior y educación superior los han desdeñado. En el aula en general se le pide al estudiante que participe oralmente en clase; es decir, se le solicita que sea un enunciador oral académico. Sin embargo la escuela padece de una doble miopía: por un lado, no les permite ver que han perdido de vista la importancia de la narración en actos académicos como la conferencia, la exposición oral en clase, la charla, el discurso, la entrevista, el debate y el diálogo; muchas veces no se toma en consideración que el aula debe dotar al estudiante de competencias para las distintas situaciones de comunicación que enfrenten en su relación en la otredad; por otro, no existe una planificación y gradación de la enseñanza de la oralidad, es más ni siquiera existe la oralidad como aprendizaje de la enseñanza.
} 
Otra habilidad comunicativa importante para incentivar los procesos de aprendizaje es la escritura. De ella podemos decir que lleva implícito un conjunto de habilidades cognoscitivas que, a diferencia de la lectura o de la oralidad, tienen que adquirirse de forma planificada y sistemática, considerando la diferencia entre el producto que emana de la escritura y el proceso de construcción del mismo. La composición escrita implica una serie de habilidades cognitivas, por lo que escribir no sólo exige pensar, sino que es también un medio para pensar (lo mismo ocurre con la oralidad), pues funciona como un auxiliar de la memoria y permite desarrollar actividades cognitivas como identificar, comparar, analizar, diferenciar, clasificar, razonar, inferir, sintetizar, entre otras (Rojas, 2002).

La oralidad como la escritura exige un nivel de comprensión tan complejo el uno como el otro. Su importancia estriba en que las personas que carecen de este tipo expresión ven reducido su trabajo profesional, su vida académica y sus vínculos sociales con la comunidad en la que convergen, al mismo tiempo que empobrecen sus niveles simbólicos y de significado; por ello, “[...] en el proceso de enseñanzaaprendizaje hay que tener en cuenta la lengua en todas sus manifestaciones, lo que le permite al docente elevarse desde la realidad lingüística inmediata, próxima y real que utiliza el estudiante, como usuario de la lengua, hasta los niveles en los que el alumno perciba que la lengua puede convertirse en una obra de arte" (Reyzábal, 2006) a través de la literatura. ${ }^{7}$

Cuando al estudiante se le enseña a ampliar sus habilidades comunicativas de expresión oral y escrita, mejora sus capacidades de aprendizaje porque tiene más herramientas para adquirir, asimilar, estructurar, retener y reproducir sus saberes (Reyzábal, 2006) y re-significarlos, ya que escribir, hablar y escuchar - como señalamos anteriormente- no sólo exige pensar, sino que es un medio para ello, pues son auxiliares para otras actividades cognitivas.

El manejo acertado de estas habilidades comunicativas conduce a dotar al alumno de herramientas para pensar y reflexionar, lo cual al mismo tiempo se reflejará en su aprovechamiento académico, ya que permitirá una mejor comprensión de los contenidos de sus unidades de aprendizaje. La carencia de estas habilidades co-

\footnotetext{
${ }^{7}$ Para ello, será importante el rescate la literatura en la enseñanza de lectura y la redacción, pues de ella se han olvidado las instituciones de educación superior.
} 
municativas puede afectar académica y emocionalmente al estudiante, pues puede conducir al individuo a sentirse "incapaz" para aprender afectando directamente su autoestima, confianza y seguridad.

En síntesis, la oralidad y la escritura implican la capacidad de saber comunicar en el terreno del saber y el saber hacer, una correlación entre determinadas teorías cognitivas y de operación de esa cognoscibilidad en la actividad humana. Pensar en este sentido conduce a pensar en qué se dice y cómo se dice, el por qué se dice y el para qué se dice, tanto en la oralidad como en la escritura, lo que condiciona el tratamiento de intenciones y finalidades comunicativas adecuadas a la situación de comunicación que se le presenta al emisor.

Es por ello que el método aprende a escuchar pensar escribir (AEPE) va a contribuir a desarrollar las habilidades específicas de aprendizaje como lo requieren los perfiles de las carreras de Contaduría y Administración de Empresas para que el rendimiento escolar sea óptimo. El objetivo de este método es dar a los estudiantes de las carreras contables-administrativas las habilidades comunicativas que les permitan disminuir su situación de reprobación y/o rezago académico, lo cual influye en la disminución de la deserción escolar, lo cual impactará positivamente en los índices de la eficiencia terminal como la obtención de grado.

En nuestra investigación se empleó un diseño cuasiexperimental de campo, de tipo longitudinal con cuatro evaluaciones (pretest, postest y seguimientos de 6 y 12 meses) para dos grupos aleatorios (uno de control y otro experimental) de estudiantes con irregularidad estudiantil. De la población de 488 estudiantes, se eligió una muestra forzada ${ }^{8}$ de 170 , que pertenecían a ambas carreras ( 88 de Contabilidad y 82 de Administración). Los 170 estudiantes fueron asignados a dos grupos ( 85 en un grupo experimental y 85 en un grupo control); dicha asignación fue realiza de acuerdo con los últimos tres dígitos que coinciden con la tabla de números aleatorios.

El material empleado se centró en los test de matrices progresivas de Raven, un test de estrategias de aprendizaje ACRA y trayectorias académicas. El test de matrices progresivas fue creado por J. C. Raven en 1938 para medir el factor general "G" de la inteligencia. Se utilizó el manual de la segunda edición ampliada (1996), escala para adultos, serie MPG con 60 problemas repartidos en cinco series (A, B,

${ }^{8}$ Muestra forzada: selección de estudiantes que tienen la condición de irregularidad estudiantil. 
C, D y E), cada una con doce elementos, con buen coeficiente de fiabilidad, $\alpha .82$ (Raven y Court, 1996). Escogimos este test porque: 1) se debe medir la inteligencia general de los alumnos ya que en esta muestra tenemos el $18.03 \%$ de alumnos con promedio menor a 7 , y así poder descartar a alumnos con coeficiente intelectual (CI) menor al término medio.

El método aprende a escuchar pensar escribir (AEPE) requiere que el alumno tenga la capacidad intelectual para comparar, formar y razonar por analogía con independencia de los conocimientos adquiridos previamente por el alumno. También tuvimos en cuenta que Sternberg (1997) considera que la totalidad de sus ítems comprenden problemas analíticos de educción de relaciones y correlaciones. Estos son procesos de pensamiento indispensables para que alumno adquiera conocimientos en el nivel universitario.

El test de estrategias de aprendizaje ACRA consta de 119 ítems, evalúan un total de 32 estrategias de aprendizaje agrupadas en cuatro grandes subescalas: adquisición, codificación o almacenamiento, recuperación y apoyo (Román y Gallego, 1994); la aplicación es colectiva y con puntuaciones globales, su coeficiente de fiabilidad es $\alpha=0.84$ (Camarero, Martin del Buey y Herrero, 2000). Se eligió este test debido a lo siguiente: 1) el test contiene escalas que miden estrategias de aprendizaje, las cuales se encuentran construidas bajo el modelo de procesamiento de menoría y percepción elaborado y actualizado por Shiffrin (2003), así como en la teoría de los niveles de procesamiento de Craik y Tulving (1975) y en las teorías acerca de la representación mental del conocimiento en la memoria; 2) el test tiene tres subescalas que pueden medir procesos cognitivos básicos y la cuarta subescala puede medir procesos meta-cognitivos; ambos niveles son indispensables para saber si el método de aprendizaje aplicado es adecuado para disminuir la irregularidad estudiantil que afecta directamente a la eficiencia terminal.

Para llevar a cabo lo anterior partimos de las siguientes hipótesis:

- En la aplicación del método aprender a escuchar pensar escribir existirá una disminución significativa en número de estudiantes con irregularidad estudiantil, $p<0.05$.

- En la aplicación del método aprender a escuchar pensar escribir existirá una aumento significativo en los resultados globales del test estrategias de aprendizaje (AGRA), $p<0.05$. 
- En la aplicación del método aprender a escuchar-pensar-escribir existirá una disminución significativa en número de materias reprobadas por estudiante, $p<0.05$.

El tiempo en que se realizó el presente estudio fue de agosto de 2009 a diciembre de 2010. Las acciones que se llevaron a cabo fueron las siguientes:

1. Selección de la muestra, se escogieron a todos los estudiantes de las carreras de Contaduría y Administración de Empresas que tiene irregularidad estudiantil, $\mathrm{n}=170$.

2. Se realizó un estudio de 170 trayectorias académicas de la muestra para saber su estatus como alumno irregular y su número de materias reprobadas por semestre.

3. Condiciones éticas. Los estudiantes recibieran iguales condiciones de procedimientos como lo menciona las declaraciones de Helsinki de Tokio y la Norma Oficial NOM-028-SSA2-1999, establecida por la Secretaría de Salud y Asistencia (1999).

4. Se aplicó el formato de consentimiento y responsabilidad para ser firmada, la cual tuvo un uso confidencial. Requisito para ingreso los procedimientos de investigación.

5. Se aplicó el test de Raven para conocer el coeficiente intelectual (CI) de los estudiantes y así poder descartar a estudiantes con CI deficiente, $\mathrm{n}=170$.

6. Se aplicó el pretest; cuestionario de ACRA para evaluar las estrategias de aprendizaje que tienen los estudiantes de la muestra $n=170$.

7. Aplicación el método aprende a escuchar pensar escribir (AEPE) en 85 estudiantes del grupo experimental.

8. Aplicación de postest, test de ACRA para conocer impacto que tuvo el método AEPE, y en seguimientos de 6 y 12 meses para observar el sostenimiento que tuvo el método AEPE.

9. Se compararon los resultados a $n=170$ en número de materias reprobadas (trayectorias académicas) en evaluaciones pretest, postest y seguimiento de 6 y 12 meses para observar el impacto y el sostenimiento que tiene método AEPE.

\section{Intervención}

El propósito general fue comprender la importancia de la lectura, la escritura y la oralidad en el proceso de construcción del conocimiento al mismo tiempo que 
ejerciten estas habilidades comunicativas para su aprendizaje y el desarrollo de su pensamiento. El tiempo total de intervención fue de 80 horas, repartidas en 40 sesiones, dos sesiones por semana con dos horas cada una.

\section{Sesión 1: la lectura}

El propósito fue que los participantes revisarán los diferentes tipos de lectura y practicarán la misma con el fin de aprender estrategias lectoras, actividades realizadas de acuerdo con Reyzabal, 2006 y Cassany, 2000. Para ello se realizaron las siguientes actividades:

1. Identificar los problemas con la lectura, la escritura y la oralidad.

2. Presentación teórica y gráfica de los modelos textuales: descripción, narración, explicación o exposición y argumentación (Calsamiglia y Tuson, 2004).

3. Presentación teórica y gráfica de los textos literarios, expositivos y científicos (Calsamiglia y Tuson, 2004).

4. Presentación teórica y gráfica de los tipos de lectura: exploratoria, de comprensión y analítica (Serafini, 2006).

\section{Sesión 2: la lectura}

El propósito aquí fue que los participantes revisarán los textos expositivos y científicos con el fin de aprender estrategias lectoras ante este tipo de discursos, actividades realizadas de acuerdo con Serafini, 2006. Para ello se realizaron las siguientes actividades:

1. Elaboración de una lectura exploratoria y una lectura analítica con textos literarios (Lomas y Tusón, 2009).

2. Lectura exploratoria y lectura analítica de un texto científico (Lomas y Tusón, 2009).

\section{Sesión 3: la escritura}

El propósito de esta sesión fue que los participantes comprendieran y practicaran los diferentes modelos textuales que la escritura exige (Calsamiglia y Tuson, 2004). Para ello se realizó la construcción de modelos textuales. 
Sesión 4: la escritura

El propósito fue que los participantes comprendieran y practicaran las operaciones textuales, actividades realizadas de acuerdo con Cassany, 2000. Para ello se realizó la elaboración de un texto que contuviera las operaciones textuales aprendidas.

Asimismo, otro propósito fue que los participantes comprendieran y practicaran la oralidad (hablar y escuchar), actividades realizadas de acuerdo con Reyzábal, 2006. Para llevar a cabo esto se realizó lo siguiente:

1. Presentación oral del trabajo académico.

2. Evaluación grupal de la exposición oral con base en el protocolo de oralidad.

\section{Resultados}

Se utilizó estadística descriptiva para la presentación y análisis de distribuciones de los datos de interés de la muestra (ver cuadro 1 ).

\section{Cuadro 1}

\section{Características de la muestra}

\begin{tabular}{|c|c|c|c|c|c|}
\hline \multirow{3}{*}{\begin{tabular}{|c|} 
Variables demográficas \\
Rango de edad \\
\end{tabular}} & \multicolumn{2}{|c|}{ Socio-estudiantes } & \multirow{2}{*}{ Variable académicas } & \multicolumn{2}{|c|}{ estudiantes } \\
\hline & \multicolumn{2}{|r|}{$\mathrm{n}=170$} & & & \\
\hline & $\mathbf{n}$ & media & Nivel académico & $\mathbf{n}$ & $\%$ \\
\hline $18-20.11$ años & 51 & \multirow{5}{*}{$\begin{array}{l}24.3 \\
\text { años }\end{array}$} & primer semestre & 45 & 28.9 \\
\hline $21-24.11$ años & 63 & & tercer semestre & 62 & 27.2 \\
\hline 25 - 26.11 años & 34 & & quinto semestre & 40 & 30.9 \\
\hline $27-28.11$ años & 14 & & séptimo semestre & 17 & 15.7 \\
\hline 29.- más años & 8 & & noveno semestre & 6 & 18.0 \\
\hline Género & n & $\%$ & Carrera & $\mathbf{n}$ & $\%$ \\
\hline femenino & 67 & 45.29 & Contaduría & 88 & 51.77 \\
\hline masculino & 93 & 54.70 & Administración & 82 & 48.23 \\
\hline Estado civil & $\mathbf{n}$ & $\%$ & Promedio anual & $\mathbf{n}$ & $\%$ \\
\hline soltero & 105 & 61.74 & $5-5.9$ & 11 & 6.4 \\
\hline casado & 33 & 19.41 & $6-6.9$ & 77 & 45.29 \\
\hline unión libre & 25 & 14.70 & $7-7.9$ & 45 & 26.47 \\
\hline divorciado & 7 & \multirow[t]{2}{*}{4.1} & $8-8.9$ & 29 & 17.05 \\
\hline & & & $9-9.9$ & 8 & 4.7 \\
\hline Ocupación extra & $\mathbf{n}$ & $\%$ & Turno académico & $\mathbf{n}$ & $\%$ \\
\hline trabaja & 65 & 38.23 & matutino & 65 & 38.23 \\
\hline Realiza otros estudios & 23 & 13.52 & vespertino & 105 & 61.77 \\
\hline Realizan servicio social & 10 & 5.88 & Materias reprobadas & $\mathbf{n}$ & $\%$ \\
\hline Prácticas profesionales & 2 & \multirow[t]{2}{*}{1.17} & $1-2$ & 134 & 78.81 \\
\hline & & & $3-4$ & 36 & 21.17 \\
\hline
\end{tabular}


De igual manera, se utilizó la prueba t de student de variables independientes para dos muestras, existiendo igualdad de varianza; por ende, no existió diferencia significativa entre los dos grupos $\mathrm{p}>0.05$. Asimismo, se realizó la prueba de ANOVA para hacer comprobación de hipótesis, donde se observó que existe diferencia significativa $(\mathrm{p}<0.05)$ entre el grupo experimental y el grupo de control en número de alumnos con irregularidad estudiantil. Se acepta la hipótesis verdadera sólo en evaluaciones seguimiento de seis y doce meses (ver cuadro 2).

\section{Cuadro 2}

Prueba de ANOVA entre grupo experimental y grupo de control en número de alumnos con irregularidad estudiantil

\begin{tabular}{|c|c|c|c|c|c|}
\hline Grupos & $\begin{array}{c}\text { Variable } \\
\text { dependiente }\end{array}$ & Evaluaciones & & grupo & \\
\hline \multirow{3}{*}{$\begin{array}{c}\text { Control } \\
\text { experimental }\end{array}$} & \multirow{3}{*}{$\begin{array}{c}\text { Irregularidad } \\
\text { estudiantil }\end{array}$} & \multirow[b]{2}{*}{$\begin{array}{c}\text { Seguimiento } \\
\text { seis meses }\end{array}$} & $\mathrm{gl}$ & $\mathrm{F}$ & $\mathrm{p}<0.05$ \\
\hline & & & 158 & 7.863 & 0.038 \\
\hline & & $\begin{array}{l}\text { Seguimiento } \\
12 \text { meses }\end{array}$ & 114 & 4.205 & 0.046 \\
\hline
\end{tabular}

También se realizó una prueba de ANOVA para la comprobación de hipótesis, en la cual se observó que existe diferencia significativa $(\mathrm{p}<0.05)$ entre el grupo control y el grupo experimental en resultados de globales del test de estrategias de aprendizaje ACRA. Se aceptó la hipótesis verdadera sólo en la evaluación postest (ver cuadro 3).

\section{Cuadro 3}

Prueba de ANOVA entre grupo control y experimental en resultados de globales del test de estrategias de aprendizaje ACRA

\begin{tabular}{cccccc}
\hline Grupos & $\begin{array}{c}\text { Variable } \\
\text { dependiente }\end{array}$ & Evaluaciones & \multicolumn{3}{c}{ Entre grupos } \\
\hline $\begin{array}{c}\text { Control y } \\
\text { experimental }\end{array}$ & $\begin{array}{c}\text { Estrategias } \\
\text { aprendizaje }\end{array}$ & Postest & 165 & 10.27 & 0.043 \\
\cline { 3 - 6 }
\end{tabular}

Además, se implementó la prueba t de student y prueba F de Fisher para dos muestras independientes con el número de materias reprobadas al semestre para saber si existía una disminución significativa en los grupos. Se observó que el grupo experimental tuvo disminución tanto en seguimiento de seis doce meses; en el grupo control sólo se dio en seguimiento de doce meses (ver cuadro 4). 


\section{Cuadro 4}

Prueba $t$ de student y prueba $\mathbf{F}$ de Fisher para dos muestras Independientes en número de materias reprobadas al semestre

\begin{tabular}{|c|c|c|c|c|c|c|c|}
\hline \multirow{2}{*}{ Grupo } & \multirow{2}{*}{$\begin{array}{c}\text { Variable } \\
\text { dependiente } \\
\text { Número de } \\
\text { materias } \\
\text { reprobadas } \\
\text { al semestre }\end{array}$} & \multirow[t]{2}{*}{ Evaluación } & \multicolumn{3}{|c|}{ Prueba t Student } & \multicolumn{2}{|c|}{$\begin{array}{c}\text { Prueba F } \\
\text { Fisher } \\
\text { Entre grupos }\end{array}$} \\
\hline & & & $\mathrm{t}$ & gl & $\mathrm{p}>0.05$ & $\begin{array}{l}\text { Leven } \\
\mathrm{F}\end{array}$ & $\mathrm{p}<0.05$ \\
\hline \multirow{2}{*}{ Experimental } & $1-2$ & $\begin{array}{l}\text { Seguimiento seis } \\
\text { meses }\end{array}$ & 1.34 & 62 & 0.653 & 2.650 & 0.042 \\
\hline & $3-4$ & $\begin{array}{c}\text { Seguimiento doce } \\
\text { meses }\end{array}$ & 0.75 & 11 & 0.899 & 3.087 & 0.039 \\
\hline Control & $1-2$ & $\begin{array}{c}\text { Seguimiento doce } \\
\text { meses }\end{array}$ & 1.76 & 52 & 0.387 & 1.978 & 0.049 \\
\hline
\end{tabular}

\section{Discusión}

El trabajo se centró en mejorar las habilidades de aprendizaje medido a través de disminución de la irregularidad estudiantil porque es un índice que marca a los alumnos que pueden tener deserción escolar o bajo rendimiento académico en su eficacia terminal. Para lograr esta disminución se propuso el método aprende a escuchar-pensar-escribir (AEPE), puesto que este método agiliza procesos básicos cognitivos a través de la enseñanza de las competencias de la oralidad y la escritura, elementos que se vienen enseñando desde la primera infancia.

Es conocido por todos que el estudiante universitario se encuentra perfectamente capacitado para afrontar sus estudios con éxito, dependiendo de su esfuerzo (Castelló y Monereo, 1999; González, Valle, Rodríguez y Piñeiro, 2002; Nist y Simpson, 2000). "Se atribuye a la educación, la misión de permitir a todos los ciudadanos el hacer fructificar todos sus talentos y todas sus capacidades de creación [...]" (Martín, García, Torbay y Rodríguez, 2007).

Existen diversos estudios, como el realizado por la UNESCO en 2001 y la encuesta realizada por Conaculta en 2010, donde se observa el rezago de hábitos de lectura y las consecuencias de los mismos en la preparación que tienen nuestros estudiantes universitarios. Estos dos puntos de vista son opuestos de lo que debe ser y lo que es. Esta reflexión es demostrada en nuestros resultados, pues se observa 
en el postest que existe diferencia entre el grupo control y el grupo experimental con relación en la disminución de alumnos con irregularidad estudiantil. Los datos se vuelven estadísticamente significativos en las evaluaciones realizadas después de seis y doce meses. Con ello se demuestra que la enseñanza del lenguaje oral y escrito en la universidad es imprescindible, a pesar de que parte del profesorado piense lo contrario y muchas veces se pregunten: ¿para qué enseñar a hablar al estudiante cuando éste realiza esta actividad de manera efectiva sin enseñanza alguna?, o cuestione ¿se puede enseñar a hablar? Este tipo de interrogantes demuestra que hablar bien o hablar mejor no es una habilidad valorada en el proceso educativo en la enseñanza de la lengua. ${ }^{9}$

Como lo afirmamos anteriormente, no todas las manifestaciones comunicativas orales son "naturales", pues algunas requieren ser aprendidas con el mismo grado de seriedad, calidad y complejidad que exige, por ejemplo, la escritura o la lectura.

En nuestro estudio, el impacto de la intervención se pudo constatar en las 32 estrategias que mide el test de ACRA porque se observó una diferencia significativa en la evaluación postest entre ambos grupos. El test de ACRA es una escala que mide estrategias de aprendizaje de acuerdo con niveles de procesamiento de Craik y Tulving (1975), por lo que se puede inferir que los estudiantes modificaron estrategias de aprendizaje que contribuyeron a disminuir la irregularidad estudiantil. Otros autores se refieren a ella como aprender a aprender (Pozo, Monereo y Castelló, 2001), aludiendo a las capacidades metacognitivas del pensamiento evaluada en la subescala de apoyo de aprendizaje.

En nuestros resultados también se pudo observar que existe una disminución significativa de materias reprobadas en un seguimiento de seis y doce meses; también es importante señalar que el grupo control mostró dicha disminución, pero en cuanto a la norma reglamentaria de estatutos universitarios para que el alumno no obtuviera baja académica.

\footnotetext{
9“'Los chicos y chicas castellanohablantes de 16 o 17 años [...] han sido víctimas de una enseñanza ineficaz y gramaticalista, que ha sacrificado la utilidad y la necesidad de la comunicación oral cotidiana a la teoría lingüística; que ha sustituido la práctica expresiva en el aula por la memorización y la gramática escrita y el estudio de la literatura, y las tradiciones culturales. Estos alumnos se lamentarán durante mucho tiempo — como también lo haremos los profesores y toda la sociedad—de este gran error" (Daniel Cassany et al., 2000, p.137).
} 
En síntesis, la enseñanza de habilidades comunicativas en el aula - en cada una de las unidades de aprendizaje que el estudiante curse en su estancia universitariafavorecerá el rendimiento escolar y mejorara la tarea intelectual que la universidad exige, pues será un mecanismo para enseñar y ejercitar las competencias comunicativas en aquellos sujetos que aún no han desarrollado estas habilidades, con lo que mejorarán sus posibilidades de trabajo y estudio. Esto permitirá a nuestros educandos, entre otras cosas, saber cómo controlar su propio aprendizaje, cómo diagnosticar sus puntos fuertes y débiles como alumno, cómo describir su estilo o estilos de aprendizaje, conocer en qué condiciones aprende mejor, cómo aprender de la experiencia de cada día y cómo superar las dificultades que enfrenta cotidianamente en su vida académica. Escribir, hablar y escuchar no sólo exige pensar, sino es un medio para pensar.

Ante este fenómeno multifactorial, para futuros estudios se puede hacer un análisis cualitativo de cuáles son los factores o variables que influyen en cada uno de los procesos de pensamiento para que disminuya la irregularidad estudiantil.

\section{Referencias}

Allgood, W.P., V.J. Risko, M.C. Álvarez y M.M. Fairbanks (2000). Factors that influence study. Handbook of college reading and study strategies research. R. F. Flippo y D. C. Caverly, eds. New York: Lawrence Erlbaum Associates: 201-219. Disponible en: http://acapsi.com/estrategias_de_aprendizaje_y_rendimiento_academico_en_estudiantes_universitarios.html

Argudín, F. y M. Luna (2006). Aprender a pensar leyendo bien. México: Paidós.

Arguelles, J.D. (2007). Conferencia Influir y leer. I Congreso Departamental de Lectura, La lectura como camino a la equidad. Medellín. Disponible en: http://www.bibliotecanacional.gov.co/index.php?idcategoria=36758

Buela-Casal, G. y A. Castro (2008). Criterios y estándares para la obtención de la Mención de Calidad en Programas de Doctorado: evolución a través de las convocatorias. International Journal of Psychology and Psychological Therapy 8: 127-136. Disponible en: http://www.redalyc.uaemex.mx/redalyc/pdf/560/56080111.pdf 
Camarero, S., F. Martín del Buey y D. Herrero (2000) Estilos y estrategias de aprendizaje en universitarios. Psicothema 12 (4): 615-622. Disponible en: http://www.psicothema.com/pdf/380/pdf

Cassany, D., M. Luna y G. Sanz (2000). Enseñar Lengua. 6a. ed., Barcelona: Grao.

Calsamiglia, H. y A. Tusón (2004). Las cosas del decir. Manual de análisis del discurso. México: Paidós.

Castelló, M. y C. Monereo (1999). El conocimiento estratégico en la toma de apuntes: un estudio en la educación superior. Infancia y Aprendizaje 88: 25-42. Disponible en: http://www.dialnet.unirioja.es/servlet/articulo?codigo $=48622$

Consejo Nacional para la Cultura y las Artes (2010). Encuesta Nacional de hábitos, prácticas y consumo culturales, Comparativo estatal de resultados básicos. México: Conaculta. Disponible en: http://www.conaculta.gob.mx/recursos/ encuesta_nacional/2010/Comparativo_Estados_2010.pdf

Craik F. y E. Tulving (1975). Depth of Processing and the Retention of Words in Episodic Memory: Journal of Experimental Psycholog, 104 (3): 268-294. Disponible en: http://www.ballarat.edu.au/ard/bssh/school/hp502/Craik\%2 0and\%20Tulving,\%201975.pdf

Chain, R. (1999). La tutoría. Una alternativa para contribuir a abatir los problemas de deserción y rezago en la educación superior. México: ANUIES. Disponible en: http://www.anuies.mx/servicios/d_estrategicos/libros/ lib42/17.htm

Díaz de Cossio, R. (1998). Programas Institucionales de Tutoría. Una propuesta de la ANUIES para su organización y funcionamiento en las Instituciones de Educación Superior. Asociación. México: ANUIES. Disponible en: http:// www.anuies.mx/servicios/p_anuies/publicaciones/revsup/127/01b.html

Donolo, D., A. Chiecher y M. Rinaudo (2004). Estudiantes, estrategias y contextos de aprendizajes presenciales y virtuales. Conferencia realizada el 12 de mayo de 2004. Disponible en: http://www. virtual.unlar.edu.ar/jornadas-conferencias-seminarios/jornada-interprov-ead/2003_3ra/ponencias-y-trans/est-cog-y-estr-apr.pdf 
González, C.R., A. Valle, S. Rodríguez e I. Piñeiro (2002). Autorregulación del aprendizaje y estrategias de estudio. Estrategias de aprendizaje. Concepto, evaluación e intervención. J. González-Pineda, J. Núñez, L. Álvarez y E. Soler, coords. Madrid: Pirámide: 17-38.

Gutiérrez, V.A. y G.R. Montes de Oca (2004). La importancia de la lectura y su problemática en el contexto educativo universitario. El caso de la Universidad Juárez Autónoma de Tabasco (MÉXICO). Revista Iberoamericana de Educación (28): 25-40.

Hauy, M.E. (2008). Leer literatura. México: Encuentro.

Martín, E., L. García, A. Torbay y T. Rodríguez (2007). Estructura factorial y fiabilidad de un cuestionario de estrategias de aprendizaje en universitarios: CEA-U. Anales de Psicología (23): 1-6. Disponible en: http://www.redalyc. uaemex.mx/redalyc/pdf/167/16723101.pdf

Lomas, C. y A. Tusón (2009). Enseñanza del lenguaje, emancipación, comunicación y educación crítica. México: Edere.

Moyano, M., C. Delgado y G. Buela-Casal (2006). Análisis de la productividad científica de la Psiquiatría española a través de las tesis doctorales en la base de datos TESEO (1993-2002). Internacional Journal of Psychology and Psychological Therapy (6): 11-120. Disponible en: http://www.ijpsy. com/volumen6/num1/133.html

Nist, S. y M. Simpson (2000). College Studying. Handbook of Reading Research, (2). M. Kamil, M., Mosenthal, P., Pearson, P. y Barr, R. Nueva York: Longman. pp. 645-666.

Pintrich, P., (2004). A conceptual framework for assessing motivation and self-regulated learning in college students. Educational Psychology Review, 16: 385-407. Disponible en: http://www.mendeley.com/research/a-conceptual-frameworkfor-assessing-motivation-and-selfregulated-learning-in-college-students/

Universidad Autónoma del Estado del México (2010). Documento interno. Coordinación de Servicios escolares, Departamento de Control Escolar, Plantel Centro Universitario del Valle de México. 
Paul, Carlos y Ángel Vargas (2001). México inmerso en el analfabetismo funcional, una catástrofe silenciosa. La Jornada, 15 enero: 1-2.

Pozo, J., C. Monereo y M. Castelló (2001). El uso estratégico del conocimiento. Desarrollo psicológico y educación. Tomo II. C. Coll, J. Palacios y A. Marchessi, comps. Madrid: Alianza.

Programas Institucionales de Tutoría. (2002). Una propuesta de la ANUIES para su organización y funcionamiento en las Instituciones de Educación Superior. México: ANUIES.

Raven, J.C. y J.H. Court (1996). Raven. Matrices progresivas. 2a. ed., Madrid: TEA.

Reyzábal, M.V. (2006). La comunicación oral y su didáctica. 6a. ed., Madrid: La Muralla.

Roales-Nieto, J. G. (2007). Análisis de la movilidad del alumnado en los estudios universitarios de Psicología en España. International Journal of Psychology and Psychological Therapy, 7 (1): 73- 117. Disponible en: http://redalyc. uaemex.mx/pdf/560/56070106.pdf

Rojas, R. (2002). El arte de hablar y de escribir. Experiencias y recomendaciones. México: Plaza y Valdés.

Román, J.M. y S. Gallego (1994). Escalas de estrategias de aprendizaje. (ACRA). México: Manual Moderno.

Serafini, M. T. (2006). Cómo se estudia. Organización del trabajo intelectual. México: Ariel.

Shiffrin, M.R. (2003). Modeling memory and perception. Cognitive Science (27): 341-378. Disponible en: http:maplab.cogs.idiana.edu/pubs/Shiffrin 03.pdf

Sternberg, R. J. (1997). La creatividad en una cultura conformista: un desafío a las masas. Barcelona: Paidós.

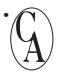

